\title{
Muller's ratchet in random graphs and scale-free networks
}

\author{
Paulo R. A. Campos, ${ }^{1, *}$ Jaime Combadão, ${ }^{2}$ Francisco Dionisio, ${ }^{2,3}$ and Isabel Gordo ${ }^{2, \dagger}$ \\ ${ }^{1}$ Departamento de Física e Matemática, Universidade Federal Rural de Pernambuco, Dois Irmãos 52171-900, Recife-PE, Brazil \\ ${ }^{2}$ Instituto Gulbenkian de Ciência, Rua Quinta Grande, 6, Apartado 14, P-2781-901 Oeiras, Portugal \\ ${ }^{3}$ Dep Biol Vegetal, Faculdade de Ciências, Universidade de Lisboa, Campo Grande, Ed.C2 PT-1749-016 Lisboa, Portugal
}

(Received 3 February 2006; revised manuscript received 1 September 2006; published 4 October 2006)

\begin{abstract}
Muller's ratchet is an evolutionary process that has been implicated in the extinction of asexual species, the evolution of mitochondria, the degeneration of the Y chromosome, the evolution of sex and recombination and the evolution of microbes. Here we study the speed of Muller's ratchet in a population subdivided into many small subpopulations connected by migration, and distributed on a network. We compare the speed of the ratchet in two distinct types of topologies: scale free networks and random graphs. The difference between the topologies is noticeable when the average connectivity of the network and the migration rate is large. In this situation we observe that the ratchet clicks faster in scale free networks than in random graphs. So contrary to intuition, scale free networks are more prone to loss of genetic information than random graphs. On the other hand, we show that scale free networks are more robust to the random extinction than random graphs. Since these complex networks have been shown to describe well real-life systems, our results open a framework for studying the evolution of microbes and disease epidemics.
\end{abstract}

DOI: 10.1103/PhysRevE.74.042901

PACS number(s): 87.17.Ee, 87.16.Ac, 05.40.-a

The accumulation of deleterious mutations by the loss of the fittest individuals in an asexual population is known as Muller's ratchet [1]. Briefly the process works as follows: as most mutations are deleterious an effectively infinite population develops an equilibrium between mutation and natural selection. At this equilibrium the expected number of individuals free of deleterious mutations is given by $n_{0}$ $=N \exp (-U / s)$, where $N$ is the population size, $U$ the mutation rate, and $s$ is the selection coefficient. This is classically referred to as least loaded class. If it so happens that this class is small and due to the fact that any natural population is finite, stochastic effects become important and the class may be lost. As there is no sex, this loss is irreversible and a click of the ratchet occurs [2]. Successive clicks result in the continuous decline in mean fitness of the population. This process constitutes one of the theories invoked to explain the evolution of sex and sex chromosomes [3-5], of mitochondria genomes [6], and the extinction of small asexual populations [7]. It has also been suggested to play a role in the evolution of microbes and virus [8]. Several properties of the ratchet have been studied under the simplistic assumption that populations are unstructured [9-14]. One, that has been the subject of intense research, is the speed of the ratchet. Analytical expressions for its speed, valid over every parameter range, have been difficult to obtain, and there are only approximations valid under specific conditions. Although the process involves only three evolutionary forces in unstructured populations: mutation, selection, and drift; its quantification has constituted an extraordinary theoretical problem. From simulations and some analytical approximations it is known that in populations without structure the ratchet turns very fast when $N \exp (-U / s)<1$ and its speed becomes of little biological significance when $N \exp (-U / s) s>10$ [15].

\footnotetext{
*Electronic address: prac@ufrpe.br

†Electronic address: igordo@igc.gulbenkian.pt
}

However the vast majority of species are to some extent subdivided into small populations where individuals compete with other individuals locally [16-18]. So it is important to understand the role of population subdivision on this process. There are two commonly studied models of population subdivision: the stepping-stone model [18] and the island model [16]. Recently we studied the ratchet in a modified version of the stepping-stone model by introducing long range links between the subpopulations (small-world network) [19] while keeping the mean connectivity constant [20]. Briefly we saw that population subdivision increases the speed of the ratchet. Motivated by the pioneer works that reveal that reallife networks exhibit a power law distribution for the connectivity between their nodes [21-23], here we address the problem of mutation accumulation in both scale-free networks [21] and random graphs (Erdös and Rényi model [24]). Both the importance of spatial structure in pathogen transmission and the role of the ratchet in microbial evolution has been recently recognized [8,25-30].

We consider the evolution of subdivided populations of asexual haploid organisms, such as many bacteria. We assume nonoverlapping generations and the following life cycle: migration, mutation, and selection. The population is subdivided into $D$ demes (corresponding to the nodes of the network), each with $N_{d}$ individuals. The total number of individuals, $N_{t}=D N_{d}$, is constant. In order to model migration we do as follows. Each deme $i$ of a given network is connected to $k_{i}$ other demes. In the case of random graphs the connectivities $k_{i}$ are distributed according to a Poisson distribution of mean $z$, which denotes the mean connectivity of the graph, while in scale-free networks the distribution of connectivities obeys a power law $P(k) \propto k^{-\gamma}$, with $\gamma=3$. Each edge of the network connects two demes that exchange migrants at a mean rate $m$. We have assumed bidirectional migration. We produce a new generation of individuals by taking the following steps: we estimate the number of migrants in each deme by sampling from a Poisson distribution with 

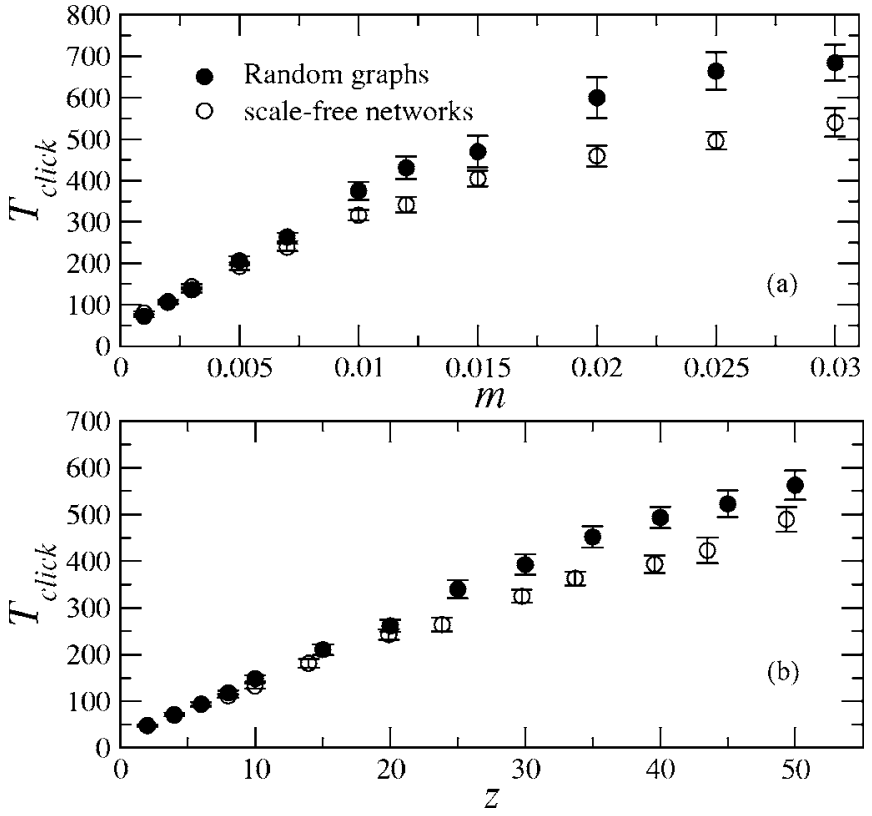

FIG. 1. Comparison of scale free and random graph networks. (a) with migration, $m$. (b) with the average connectivity of the network, $z$. The parameter values are $D=500, N_{d}=50, U=0.1$, $s=0.02$ in (a) $z=10$ and in (b) $m=0.003$.

mean $N_{d} m k_{i}$, where $k_{i}$ is the number of demes that are connected to the deme in question. The individuals that migrate are sampled at random, without replacement, from the original deme and added to the recipient demes. After migration, mutation and selection occurs. For each deme a random individual is picked and is subject to new deleterious mutations which are Poisson distributed with mean $U$. Every mutation will cause the same decrease in fitness, $s$, and an individual survives according to its fitness. We assume multiplicative fitness: $w_{j}=(1-s)^{j}$, where $j$ is the number of deleterious mutations an individual carries. In each generation, we count the number of individuals that have the lowest number of deleterious mutations in the whole population. If this least loaded class is lost, a click of the ratchet occurs. The mean time between clicks of the ratchet $T_{\text {click }}$ can then be calculated. We have made 25 runs for each set of parameters.

We start comparing the two topologies by analyzing different migration rates in a case where the ratchet is expected to click in the corresponding undivided population. Figure 1(a) shows the results for a population with $D=500$ demes in the two types of networks. From Fig. 1(a) we clearly see that increasing migration slows the ratchet in both types of networks. This is expected since, as migration rate increases, the effective population size gets closer to the one in the homogeneous case. In the figure we can observe that, for very low migration rates, the mean time between clicks of the ratchet is the same for both scale free networks and random graphs. But for high values of $m$ the ratchet clicks faster in scale free networks. So, all else being equal, random graphs are more robust to the accumulation of deleterious mutations.

In Fig. 1(b) we show that the same applies for a fixed value of $m$, but increasing the mean connectivity $z$. Again for low values of $z$ both topologies have similar rates of muta-
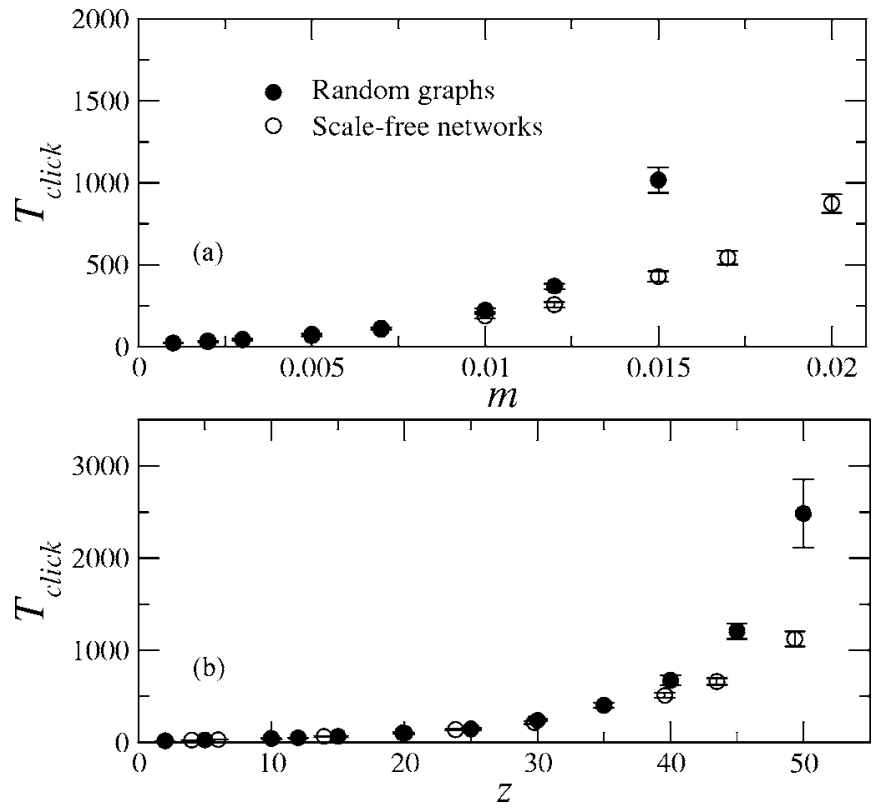

FIG. 2. Comparison of scale free and random graph networks. (a) with migration, $m$. (b) with the average connectivity of the network, $z$. The parameter values are $D=500, N_{d}=50, U=0.5, s=0.1$ in (a) $z=10$ and in (b) $m=0.003$.

tion accumulation, but, for higher mean connectivities, random graphs have a lower rate of the ratchet. For the values of $N_{t}, U$, and $s$ in Fig. 1, the mean time between clicks of the ratchet in a population without any structure is $T_{\text {click }}=1060$. It is clear that even with the highest migration and highest average connectivities simulated, none of the topologies achieved the homogeneous result. For a fixed value of $z=10$ increasing migration leads to a saturation of $T_{\text {click }}$ close to 700 for random graphs, and to 550 for scale free networks. In our algorithm one does not enable an effective number of migrants greater than $N_{d} / 2$ in a deme, which would be meaningless, since in that case the migrant population would be higher than the resident. For the case of Fig. 1(a), this occurs for $m>0.03$. When we fix $m=0.003$, but increase $z, T_{\text {click }}$ increases in both topologies, but even so does not reach the homogeneous result.

We now consider a parameter set where the ratchet does not turn in a completely homogeneous population. Figure 2 shows the results for a population with the same total size, but different parameter values of mutation and selection. Both $U$ and $s$ are 5 times higher, albeit their ratio is the same as in Fig. 1. From the figure we clearly notice an exponential growth of $T_{\text {click }}$ with both $m$ and $z$. Here the difference between random graphs and scale free networks is even more striking. In the case of random graphs with $m=0.020$ and $z=10$ we have obtained $T_{\text {click }}=3891$ (not shown), whereas in scale free networks $T_{\text {click }}=873$.

From our results we have ascertained that when the mean number of migrants per deme $N_{d} z m<1$, the ratchet turns at the same speed in both topologies. But when $N_{d} z m \gg 1$ the difference between random graphs and scale free networks emerges, with the former being less prone to genetic degeneration. In the limit $m \rightarrow 0$ all demes become isolated, and the effective population size, $N_{e} \rightarrow N_{d}$. As $m$ (or $z$ ) increases 


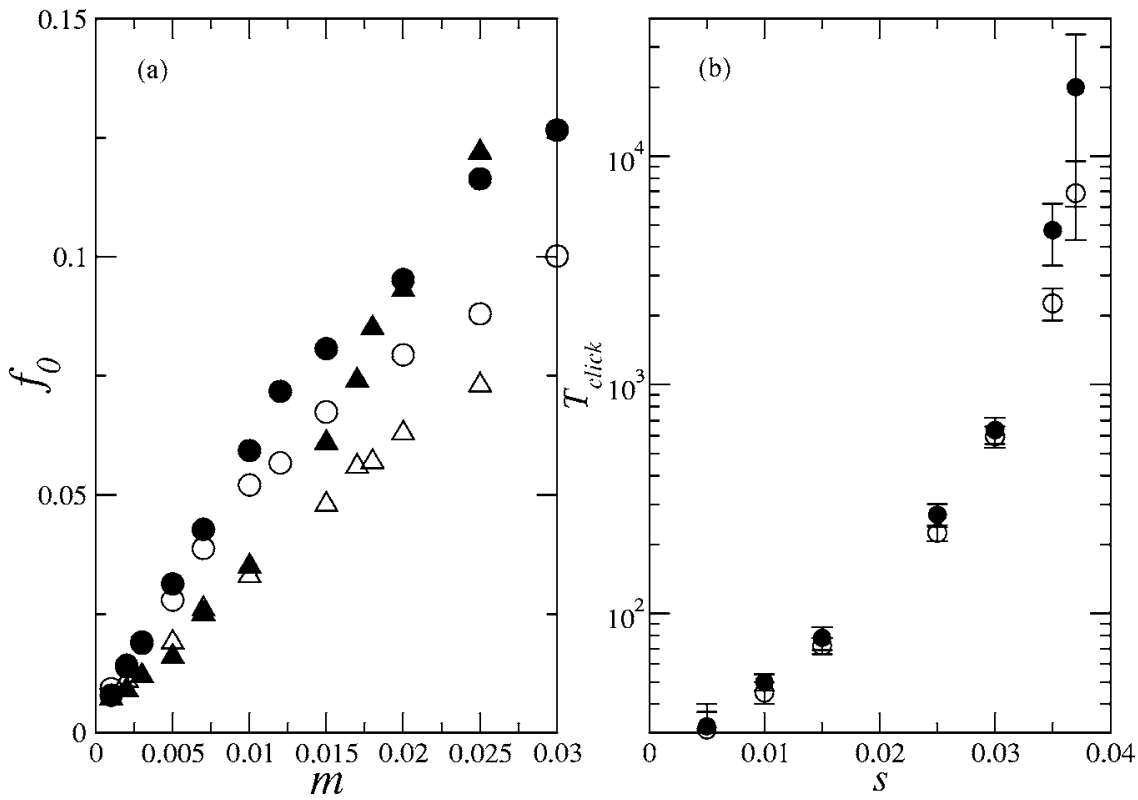

FIG. 3. (a) Fraction of demes with the least loaded class, $f_{0}$, as a function of migration rate $m$. The parameters are $D=500, N_{d}=50$, and $z=10$. Open symbols are for scale free networks and filled symbols for random graphs. For circles $U=0.1$ and $s=0.02$, for triangles $U=0.5$ and $s=0.1$. (b) The mean time between clicks of the ratchet $T_{\text {click }}$ as a function of the selective parameter $s$. The parameters are $D=200, N_{d}=50, z=10$, $m=0.005$, and $U=0.1$. Open symbols are for scale free networks and filled symbols for random graphs. the effective size increases [31], although very slowly in the regime where $N_{d} z m<1$, since the average number of migrants is too low to reduce isolation. But when $N_{d} z m>1$, the rate of increase in $N_{e}$ becomes considerable, and the efficacy of selection $N_{e} s$ is greatly increased [32]. This is why we see a more drastic increase in Fig. 2 which has a higher $s$ ) than in Fig. 1 (which has a lower $s$ ).

The properties of the different topologies can help us understand why random graphs are less prone to fitness decline. The clustering coefficient in scale free networks is higher than that in random graphs [21] mainly due to the existence of highly connected nodes (hubs). This implies that, when occurring in small number, the individuals in the least loaded class will be surely confined to a smaller number of demes than in a random graph. This leads to a smaller effective size in scale free networks compared to random graphs, and therefore a higher speed of deleterious mutation accumulation in scale free networks. If this is true then, at a given time point, the fraction of demes in which the least loaded class has not been lost will be larger in random graphs than in scale free networks. Figure 3(a) shows that this is precisely the case. In addition because the efficacy of selection against deleterious mutations depends on the effective population size, through $N_{e} s \approx 1$, the value of $s$ above which the ratchet stops will be higher in scale free networks than in random graphs. In Fig. 3(b) we compare the two topologies under the same values of $m$ and $z$ but with varying $s$. Clearly at $s$ $=0.037$ the ratchet is coming to a halt in random graphs but its speed is still considerable in scale free networks.

We have also analyzed the effect of extinction and recolonization of a deme on the speed of the ratchet. This was done by introducing, every generation, a probability $e$ that a deme goes extinct. Figure 4 shows the effect of extinction on both types of topologies. If $e$ is low, a population in a scale free network accumulates deleterious mutations at a higher rate than a population with a random graph topology, but as $e$ becomes large, the rate of the ratchet becomes similar in both topologies. In random graphs the distribution of connectivi- ties is peaked around the mean connectivity $z$, so most of the time demes with connectivity $k$ around $z$ become extinct. On the other hand, in scale free networks the extinction procedure occurs with higher probability at demes of low connectivity. In Fig. 5 we plot the conditional probability that a deme has the least loaded class in the whole population given that the deme has connectivity $k$. We compute this by averaging over time and over 1000 distinct runs. From the figure, we clearly see that in both topologies a better connected deme has a higher probability of having the least loaded class. This, together with the previous argument, allows one to infer that scale free networks are less sensitive to random extinction. Indeed their performance is comparable to that in random graphs when dealing with high extinction rates.

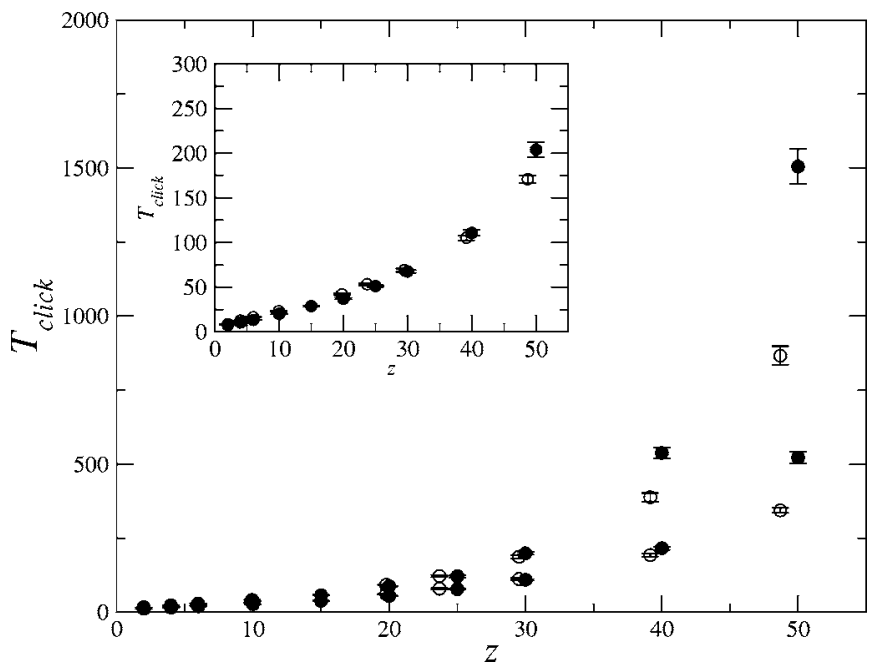

FIG. 4. Effect of random extinction on the mean time between clicks of the ratchet. The parameters are $D=500, N_{d}=50, U=0.5$, $s=0.1$, and $m=0.003$. Open symbols are for scale free networks and filled symbols for random graphs. For circles $e=0.01$, for squares $e=0.05$, and for triangles $e=0.1$ (inset). 


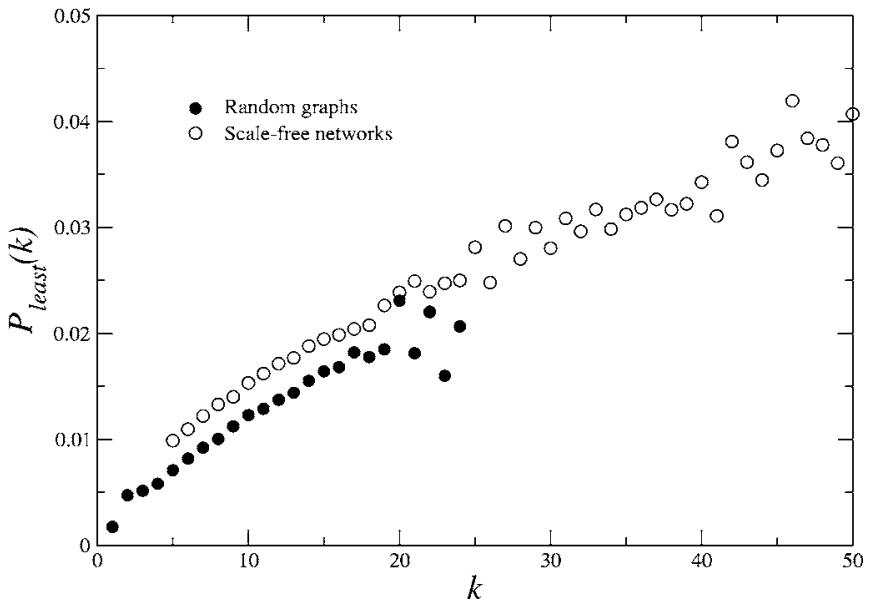

FIG. 5. The probability that a deme has the least loaded class in the whole population, $P_{\text {least }}(k)$, as a function of deme connectivity $k$, for scale free and random graphs. Parameters $D=500$, $N_{d}=50, U=0.5, s=0.1, m=0.003$, and $z=10$. Open symbols are for scale free networks and filled symbols for random graphs.

A great range of systems such as social, communication, and biological systems are well described by complex networks $[19,21-23,33]$. The understanding of the interplay between the underlying topology and the forces driving those systems is of tremendous importance $[33,34]$. One example of this, that has received a great deal of attention, is that of network epidemiology: the study of epidemic and disease spreading $[27,35,36]$, which are strictly tied to the topology of social contact networks. On this context, a striking result has arisen from the study of the classical susceptibleinfected-susceptible (SIS) epidemiological model on scale free networks: scale-free networks are more prone to spreading of diseases than random graphs and regular lattices $[27,35]$. In this kind of model the role of microbe evolution is disregarded. It is on this latter feature that we have focused here. Even though the spreading of pathogens is faster in scale-free networks than in any other topology [27,35], here we have seen that scale free networks show a higher rate of fitness decline. The life of a pathogen is dominated by continuous colonization of new hosts. Its success relies on its genome ability to deal with mutation pressure, selection arising from the host immune system and demography. Our results open a new question in the understanding of pathogen evolution: what type of network will be less advantageous for the pathogen when all the parameters of its evolutionary history are considered?

This work was supported by projects POCTI/BSE/46856/ 2002 and POCI/BIA-BDE/55758/2004 through Ciência e Tecnologia (FCT). One of the authors (P.C.) was supported by Conselho Nacional de Desenvolvimento Científico e Tecnológico $(\mathrm{CNPq})$. Three of the authors (J.C., F.D., and I.G.) were supported by Grants Nos. SFRH/BD/5235/2001, SFRH/BPD/14820/2003, and SFRH/BPD/8104/2002.
[1] J. Felsenstein, Genetics 78, 737 (1974).

[2] J. Haigh, Theor Popul. Biol. 14, 251 (1978).

[3] N. H. Barton and B. Charlesworth, Science 281, 1986 (1998).

[4] B. Charlesworth, Proc. Natl. Acad. Sci. U.S.A. 75, 5618 (1978).

[5] I. Gordo and B. Charlesworth, Genet. Res. 78, 149 (2001).

[6] M. Lynch, Mol. Biol. Evol. 13, 209 (1996).

[7] M. Lynch, R. Burger, D. Butcher, and W. Gabriel, Hereditas 84, 339 (1993).

[8] L. Chao, Nature (London) 348, 454 (1990).

[9] J. F. Fontanari, A. Colato, and R. S. Howard, Phys. Rev. Lett. 91, 218101 (2003).

[10] S. C. Manrubia, E. Lázaro, J. Pérez-Mercader, C. Escarmis, and E. Domingo, Phys. Rev. Lett. 90, 188102 (2003).

[11] I. M. Rouzine, J. Wakeley, and J. M. Coffin, Proc. Natl. Acad. Sci. U.S.A. 100, 587 (2003).

[12] A. S. Kondrashov, Genetics 136, 1469 (1994).

[13] I. Gordo, A. Navarro, and B. Charlesworth, Genetics 161, 835 (2002).

[14] I. Gordo and F. Dionisio, Phys. Rev. E 71, 031907 (2005).

[15] I. Gordo and B. Charlesworth, Genetics 156, 2137 (2000).

[16] S. Wright, Genetics 16, 97 (1931).

[17] T. Maruyama, Genet. Res. 15, 221 (1970).

[18] M. Kimura and G. H. Weiss, Genetics 49, 561 (1964).

[19] D. J. Watts and S. H. Strogatz, Nature (London) 393, 440 (1998).
[20] J. Combadao, P. R. A. Campos, F. Dionisio, and I. Gordo (unpublished).

[21] R. Albert and A.-L. Barabási, Rev. Mod. Phys. 74, 47 (2002).

[22] A.-L. Barabási and R. Albert, Science 286, 509 (1999).

[23] H. Jeong et al., Nature (London) 407, 651 (2000).

[24] P. Erdos and A. Rényi, Publ. Math., Inst. Hautes Etud. Sci. 5, 17 (1960).

[25] D. Mollison, J. R. Stat. Soc. Ser. B (Methodol.) 39, 283 (1977).

[26] A. L. Lloyd and R. M. May, Science 292, 1316 (2001).

[27] R. Pastor-Satorras and A. Vespignani, Phys. Rev. Lett. 86, 3200 (2001).

[28] C. T. Bergstrom, P. McElhany, and L. A. Real, Proc. Natl. Acad. Sci. U.S.A. 96, 5095 (1999).

[29] N. A. Moran, Proc. Natl. Acad. Sci. U.S.A. 93, 2873 (1996).

[30] I. Gordo and P. R. A. Campos, Genetica (The Hague, Neth.) 127, 217 (2006).

[31] M. C. Whitlock and N. H. Barton, Genetics 146, 427 (1997).

[32] M. Kimura, The Neutral Theory of Molecular Evolution (Cambridge University Press, Cambridge 1983).

[33] J. H. Fewell, Science 301, 1867 (2003).

[34] S. Camazine et al., Self-Organization in Biological Systems (Princeton University Press, Princeton, NJ, 2001).

[35] M. Barthélemy, A. Barrat, R. Pastor-Satorras, and A. Vespignani, Phys. Rev. Lett. 92, 178701 (2004).

[36] M. J. Keeling and K. T. D. Eames, J. R. Soc., Interface 2, 295 (2005). 